\title{
Marx, Jean-Samuel: Neues Deutschland - neues Deutschlandbild? Selbstdarstellung und Rezeption der Berliner Republik in Frankreich seit 1990, 450 S., Schöningh, Paderborn u. a. 2019.
}

\author{
Teresa Nentwig
}

Online publiziert: 13. Mai 2020

(C) Der/die Autor(en) 2020

Jean-Samuel Marx geht in seiner Doktorarbeit der Frage nach, welches Bild von sich Deutschland in Frankreich vermitteln möchte und wie dieses dort in Politik und Medien rezipiert wird. Den Untersuchungszeitraum begrenzt er auf die Zeit nach der deutschen Wiedervereinigung 1990 bis zur Eurokrise 2009/2010, wobei sein „Ausblick“ (S. 349) noch bis in das Jahr 2015 reicht. Marx' umfangreiche Quellenbasis besteht insbesondere aus öffentlichen Erklärungen deutscher Politiker_innen und Artikeln aus französischen Zeitungen wie „Le Figaro“ und „Le Monde“.

Um sein Thema einzugrenzen, konzentriert sich der Historiker ,auf bestimmte Entscheidungen, Ereignisse oder Krisen [...], die Schlüsselmomente für oder Wendepunkte in der Selbstdarstellung des deutschen Staates und/oder in ihrer Rezeption in Frankreich bilden“ (S. 17). Dies ist auch schon allein deshalb sinnvoll, weil es „kein anderes Land [gibt], über das die französischen Medien in diesem Ausmaß und in dieser Intensität berichten“ (S. 387). Die ausgewählten Wegmarken decken mehrere Politikfelder ab: neben der Innenpolitik die Außen- und Sicherheitspolitik sowie die Geschichtspolitik. Gegliedert ist die Arbeit allerdings nicht thematisch, sondern chronologisch, wobei der Autor drei Entwicklungsphasen darstellt. Nach der Einleitung (Kapitel 1) beschreibt er in Kapitel 2 die erste Phase, der er die Überschrift „Neues Deutschland - alte Bilder? Die Gegenwart der Vergangenheit. 1990-1998“ gibt. Hier analysiert Marx im Wesentlichen fünf Ereignisse, die er als zentral für die Selbstdarstellung der Bundesrepublik und deren Wahrnehmung in Frankreich ausmacht: den Golfkrieg, die Jugoslawienkrise, den Vertrag von Maastricht, die Gedenkfeier zum 50. Jahrestag des D-Days und die Militärparade auf den Champs-Élysées am 14. Juli 1994.

T. Nentwig (ه)

Georg-August-Universität Göttingen, Göttingen, Deutschland

E-Mail: teresa.nentwig@demokratie-goettingen.de 
Die zweite Phase, die Marx in Kapitel 3 seines Buches behandelt, datiert er auf die Jahre 1998 bis 2009. Sie zeichnet sich vor allem durch den Versuch des deutschen Staates aus, das eigene Land von der Vergangenheit zu emanzipieren und zu normalisieren. Um diesen Prozess darzustellen, hat Marx wieder diverse Schlüsselereignisse ausgewählt: die Wahl Gerhard Schröders zum Bundeskanzler, den Kosovokrieg, den Umzug von Parlaments- und Regierungssitz von Bonn nach Berlin, den EU-Gipfel von Nizza, den Irakkrieg, die Gedenkfeierlichkeiten zum 60. Jahrestag des D-Days, die Wahl Angela Merkels zur Bundeskanzlerin und den 20. Jahrestag des Mauerfalls.

Bei der dritten Phase, die der Autor in Kapitel 4 beschreibt, handelt es sich um einen Ausblick auf die Zeit zwischen 2010 und 2015. Hier geht Marx anhand mehrerer Fallbeispiele (darunter die Flüchtlingskrise) der Frage nach, ob es zwischen Deutschland und Frankreich eine ,schleichende Entfremdung“ (S. 349) gab. Neben den Wechselwirkungen zwischen Selbstdarstellung und Rezeption analysiert er in allen drei Phasen auch die Faktoren, die die jeweiligen Entwicklungen erklären, darunter auch äußere Einflüsse (internationale Krisen). Seine Dissertation endet mit einem Fazit, das die Ergebnisse der Arbeit zusammenfasst und bewertet.

Marx arbeitet stets gut nachvollziehbar und äußerst nuanciert heraus, dass es in der Selbstdarstellung des deutschen Staates und ihrer Rezeption in Frankreich zwei wichtige Brüche gab: zum einen 1998/1999 mit der Übernahme der Kanzlerschaft durch Gerhard Schröder, zum anderen 2009/2010 mit dem Ausbruch der Eurokrise. Kurz gesagt agierte die Bundesrepublik Deutschland nach 1998/1999 selbstbewusster und ,ging unverkrampfter mit der eigenen Vergangenheit um“ (S. 382), was sich positiv auf die Wahrnehmung des Landes in Frankreich auswirkte. Zugleich rief das erstarkte deutsche Selbstbewusstsein aber Konflikte in der Europapolitik mit Frankreich hervor. Für die Zeit nach 2009/2010 weist Marx insofern eine neuerliche Zäsur nach, als es damals zu einem Neuaufflammen der deutschlandkritischen Stimmung in Frankreich kam.

Insgesamt handelt es sich um eine sehr gut lesbare Studie, die sowohl für Historiker_innen als auch für Politikwissenschaftler_innen relevant ist. Während für erstere zum Beispiel die Abschnitte zur kollektiven Erinnerungskultur sehr interessant sind, haben für letztere beispielsweise die Passagen zu den Kanzlerwahlen Schröders und Merkels großen Mehrwert. So sind diejenigen Abschnitte sehr aufschlussreich, in denen Marx beschreibt, wie Angela Merkel von der französischen Presse anhand verschiedener Kriterien (zum Beispiel ihre Herkunft und ihr Familienstand) in den Blick genommen wurde.

Positiv hervorzuheben ist das Personenregister im Anhang der Arbeit, da es eine schnelle Recherche nach Namen erlaubt. Wiederholt macht es der Autor aber seinen Leserinnen und Lesern nicht einfach, wenn er durchaus sinnvolle Ankündigungen zur besseren Orientierung macht, diese dann aber nicht einhält. So erwähnt er zum Beispiel „vier Themenblöcke“ (S. 156), die während des Bundestagswahlkampfes 1998 die Berichterstattung und die politischen Stellungnahmen in Frankreich beherrscht hätten: „die Person Gerhard Schröder, die Lage Deutschlands 1998, die Bedeutung des Sieges Gerhard Schröders sowie dessen Einfluss auf die deutschfranzösischen Beziehungen und die europäische Integration“ (S. 157). Daran reiht sich direkt ein Absatz mit dem Titel „Die Person Gerhard Schröder“ an, sodass man 
annimmt, es folgten anschließend auch die drei übrigen Themenblöcke in separaten Kapiteln. Das ist jedoch nicht der Fall. Stattdessen schließt sich direkt der Abschnitt „Die Bedeutung und die Folgen des Wahlsieges“ an.

Leser_innenfreundlich wäre es zudem gewesen, wenn der Autor Seitenverweise eingebaut hätte. So schreibt er beispielsweise: „Auf die Frage nach der Verbindung zwischen dem Irakkrieg und der Einladung Gerhard Schröders zum D-DayGedenken wird noch eingegangen“"(S. 271), fügt aber keine Fußnote mit der entsprechenden Seite ein. Diese kleinen Monita fallen jedoch insgesamt nicht ins Gewicht, zu überzeugend ist die Studie, die einen wichtigen Beitrag zu den deutsch-französischen Beziehungen leistet.

Funding Open Access funding provided by Projekt DEAL.

Open Access Dieser Artikel wird unter der Creative Commons Namensnennung 4.0 International Lizenz veröffentlicht, welche die Nutzung, Vervielfältigung, Bearbeitung, Verbreitung und Wiedergabe in jeglichem Medium und Format erlaubt, sofern Sie den/die ursprünglichen Autor(en) und die Quelle ordnungsgemäß nennen, einen Link zur Creative Commons Lizenz beifügen und angeben, ob Änderungen vorgenommen wurden.

Die in diesem Artikel enthaltenen Bilder und sonstiges Drittmaterial unterliegen ebenfalls der genannten Creative Commons Lizenz, sofern sich aus der Abbildungslegende nichts anderes ergibt. Sofern das betreffende Material nicht unter der genannten Creative Commons Lizenz steht und die betreffende Handlung nicht nach gesetzlichen Vorschriften erlaubt ist, ist für die oben aufgeführten Weiterverwendungen des Materials die Einwilligung des jeweiligen Rechteinhabers einzuholen.

Weitere Details zur Lizenz entnehmen Sie bitte der Lizenzinformation auf http://creativecommons.org/ licenses/by/4.0/deed.de. 